\title{
Clinical significance of circulating tumor cells via combined whole exome sequencing in early stage cancer screening: A case report
}

\author{
ZIJIAN SU ${ }^{1 *}$, JIANGMAN ZHAO ${ }^{2 *}$, SHAOYING KE ${ }^{1}$, JIAN ZHANG ${ }^{1}$, \\ XIAOYU LIU ${ }^{1}$, YU WANG ${ }^{2}$, QIHONG SUN ${ }^{2}$ and QUNXIONG PAN ${ }^{1}$ \\ ${ }^{1}$ Department of Surgical Oncology, First Hospital of Quanzhou Affiliated to Fujian Medical University, \\ Quanzhou, Fujian 362000; ${ }^{2}$ Biotecan Medical Diagnostics Co., Ltd., Zhangjiang Center \\ for Translational Medicine, Shanghai 200120, P.R. China
}

Received January 5, 2018; Accepted June 27, 2018

DOI: $10.3892 /$ etm.2018.6507

\begin{abstract}
A newly-developed platform, integrating subtraction enrichment and immunostaining-fluorescence in situ hybridization (SE-iFISH), was applied to analyze the clinical significance of circulating tumor cells (CTCs) for early screening of cancer in healthy people. The present case report describes one healthy individual who accepted a CTC peripheral blood test, and $8 \mathrm{CTCs} / 7.5 \mathrm{ml}$ blood were detected. However, various conventional cancer biomarkers were all negative, including cervical cytological inspection, alpha-fetoprotein, cancer antigen (CA)-125, CA19-9, carcinoembryonic antigen (CEA), CA15-3 and human papilloma virus. To explore the origin of the CTCs, whole exome sequencing was used to analyze the CTC variation spectrum. A total of 42 mutations were associated with cancer according to analysis in COSMIC (http://cancer.sanger.ac.uk/cosmic). The results revealed a high risk of tumor in the colorectum, stomach and breast $(13,12$ and 6 variations matched, respectively). In this individual, an intestinal polyp was discovered and removed by colonoscopy. The intestinal polyp was identified to be a hyperplastic polyp by pathological diagnosis. No lesions were discovered in the stomach and breast. No CTCs were detected in this patient's blood at 1 and 6 months after removal of the lesions. This case indicates that CTC detection by SE-iFISH has potential in early stage cancer screening, and the mutation spectrum of CTC assists the tracking of its sources.
\end{abstract}

Correspondence to: Professor Qunxiong Pan, Department of Surgical Oncology, First Hospital of Quanzhou Affiliated to Fujian Medical University, 248-252 East Street, Quanzhou, Fujian 362000, P.R. China

E-mail: qunxiongpan@163.com

${ }^{*}$ Contributed equally

Key words: circulating tumor cell, whole exome sequencing, cancer screening, intestinal polyp, laser capture microdissection

\section{Introduction}

Circulating tumor cells (CTCs) are cells that detach from primary or metastatic solid tumors into the vasculature, where they can be sampled from the circulating blood stream (1). CTCs are commonly identified in the peripheral blood supply of diverse solid tumors, including breast cancer $(2,3)$, colorectal cancer (4), prostate cancer (5) etc. The migration of CTCs seems to be an early event in human carcinogenesis, even before the cancer is visible in clinical imaging. Experimental data in animal models has shown that tumors measuring less than $1 \mathrm{~mm}$ could be associated with the presence of CTCs in the bloodstream (6).

Zhangjiang Center for Translational Medicine published a series of studies of CTCs in breast cancer (7), pancreatic cancer (8) and colorectal cancer (9) using combined subtraction enrichment and immunostaining-fluorescence in situ hybridization (SE-iFISH), cooperated with other research teams. These studies demonstrated SE-iFISH CTC detection $91 \%$ positive rate for breast cancer patients (7), a sensitivity of $88 \%$ and specificity of $90 \%$ in pancreatic cancer and healthy individuals at the cutoff value of 2 cells $/ 7.5 \mathrm{ml}(8)$, and a sensitivity of $90.9 \%$ and specificity of $82.4 \%$ in colorectal cancer and healthy individuals at the cutoff value of one cell $/ 7.5 \mathrm{ml}$ (9). From these studies, CTC detection by SE-iFISH showed high sensitivity and specificity for distinguishing breast cancer, pancreatic cancer and colorectal cancer from healthy people. CTCs detection, up to date, provided potential biomarkers for screening of cancer or precancerosis.

SE-iFISH is a novel strategy to detect CTCs in blood (10), which enrich CTCs through the removal of WBCs using anti-CD45 antibody conjugated immunomagnetic particles, independent of EpCAM expression and tumor cell size. Centromere Probe 8 (CEP8), cytokeratin (CK), CD45, 4',6-diamidino-2-phenylindole (DAPI) were combined to identify CTCs. Since aneuploidy is a typical common cytogenetic abnormality in tumor cells, this feature could be exploited for CTC detection. FISH was performed on CEP8 to identify aneuploidy cells. CTCs were confirmed to be negative for CD45, positive for DAPI and either positive for PanCK staining or aneuploidy chromosome 8. Cells with characteristics of $\mathrm{CK}-/ \mathrm{CD} 45+/ \mathrm{DAPI}+/ \mathrm{CEP} 8=2$ were WBCs. 
The present case report describes a healthy female who accepted a CTC test (by SE-iFISH platform) in Quanzhou No. 1 Hospital, and the result was 8 CTCs/7.5 ml, which indicated a high risk of cancer. WES of these CTCs was performed to analyze their mutation profiles to track the lesion.

\section{Materials and methods}

Subtraction enrichment of CTCs. $7.5 \mathrm{ml}$ peripheral blood was collected by ACD anticoagulant tubes (BD Biosciences, Franklin Lakes, NJ, USA). Reagents for subtraction enrichment are Cytelligen CTC enrichment kit (Cytelligen, Inc., San Diego, CA, USA). In brief, peripheral blood (7.5 ml) was centrifuged at $800 \mathrm{x}$ g for $8 \mathrm{~min}$ at room temperature, then supernatant was discarded. The left sample was transferred to a centrifuge tube containing $3 \mathrm{ml}$ hCTC Separation Matrix. After centrifuging for $8 \mathrm{~min}$ at $450 \mathrm{~g}$, the cell suspension was collected from the buffy-coat layer. $150 \mathrm{ul}$ immunomagnetic particles conjugated anti-CD45 antibody was added into the cell suspension, which was inoculated at room temperature for $10 \mathrm{~min}$ and then placed on a magnetic stand (Promega Corporation, Madison, WI, USA) till the liquid became clear. The supernatant was pipetted off the magnetic field (non-magnetic bead-binding cell suspension) to remove leukocytes by centrifuging at $500 \mathrm{rpm}$ for $2 \mathrm{~min}$. Sedimented cells were thoroughly mixed with cell fixative and applied onto the coated CTC slides for subsequent identification.

Identification of CTCs. Reagents for CTC identification were provided by the Human Tumor Cell Identification kit (Cytelligen, Inc.). To identify aneuploidy CTCs, fluorescence in situ hybridization (FISH) and immunocytochemistry are used in combination. After a series of pre-treatment containing drying, washing and dehydration, $10 \mu 1$ of probe solution containing fluorescence-labeled alpha-satellite probes for the centromeres of the chromosome (CEP8) $(2 \mu \mathrm{g} / \mathrm{ml})$ was added and then covered with a coverslip and sealed with neutral resin. The hybridization procedure was as follows: degeneration at $75^{\circ} \mathrm{C}$ for $5 \mathrm{~min}$, followed by hybridization at $37^{\circ} \mathrm{C}$ overnight. Then the slide was rinsed with FR3 and added with monoclonal antibody anti-CD45 conjugated to Alexa Fluor 594 (Invitrogen; Thermo Fisher Scientific, Inc., Waltham, MA, USA) and anti-PanCK (CK4, 5, 6, 8, 10, 13 and 18) (Invitrogen; Thermo Fisher Scientific, Inc.) before inoculation at room temperature for $2 \mathrm{~h}$. After rinsing with PBS, the slides were mounted with mounting medium containing DAPI and photographed with a fluorescence microscope (Nikon Corporation, Tokyo, Japan). CTCs were confirmed to be negative for CD45 and either positive for PanCK staining or aneuploidy chromosome 8.

Laser capture microdissection and whole genome amplification of CTCs. The CTC fixed slide was put in ZEISS Palm MicroBeam Laser Micro Dissection System (Zeiss AG, Oberkochen, Germany). We found CTCs according to the coordinate recorded in process of CTC identification, and collected CTCs by laser micro dissection.

DNA amplification experiment of CTCs was according to the kit instruction of MalBac single cell genome amplification (YK001A/B; Yikon Genomics, Jiangsu, China). The product was quantified using DNA electrophoresis.
Whole exome sequencing. Whole exome sequencing was performed on DNA of blood, intestinal polyp and CTCs. For library construction, whole exome DNA capture was performed using Agilent SureSelect Human All ExonV5 kits following the manufacturer's instructions (Agilent Technologies, Inc., Santa Clara, CA, USA). Subsequent to the quality test, the qualified library was sequenced as 125 bp paired-end reads on an Illumina Hiseq 2500 platform (Illumina, Inc., San Diego, CA, USA).

Data analysis of whole exome sequencing. For whole exome sequencing, clean data was obtained after filtering adapter, low quality reads and reads with proportion of $\mathrm{N}>10 \%$. Reads were aligned to the reference human genome (UCSC hg19) through Burrows-Wheeler Aligner. Next, the Picard and Genome Analysis Tool kit (GATK) methods were adopted for duplicate removal, local realignment and base quality recalibration. Finally, the GATK Unified Genotyper was used for single nucleotide variation (SNV)/inDel annotation.

Somatic SNP/InDel detection was performed with Varscan 2 software. Variants were annotated using the ANNOVAR software tool. Mutations of CTCs were aligned to COSMIC database (http://cancer.sanger.ac.uk/cosmic/). The data in COSMIC is curated from number of high-quality sources and combined into a single resource. The sources include: Peer-reviewed journal articles, CGP laboratories at the Sanger Institute, TCGA data portal, the ICGC data portal, IARC p53 database. We selected cancer-related mutations and annotated relevant primary organ.

Droplet digital PCR. Each PCR (Bio-Rad Laboratories, Inc., Hercules, CA, USA) reaction system (in a total reaction volume of $20 \mu \mathrm{l}$ ) contained: $10 \mu \mathrm{l}$ Bio-Rad 2xddPCR supermix, $1 \mu \mathrm{l}$ primer and probe, $2 \mu \mathrm{l}$ DNA template (100 ng) and $7 \mu \mathrm{l} \mathrm{H}_{2} \mathrm{O}$. Droplets were generated and analyzed using the QX100 system (Bio-Rad Laboratories). Amplifications were performed using the following conditions: 1 cycle of $95^{\circ} \mathrm{C}$ for $10 \mathrm{~min}, 45$ cycles of $94^{\circ} \mathrm{C}$ for $10 \mathrm{sec}$ and $60^{\circ} \mathrm{C}$ for $45 \mathrm{sec}, 1$ cycle of $98^{\circ} \mathrm{C}$ for $10 \mathrm{~min}$, and $1 \mathrm{cycle}$ of $25^{\circ} \mathrm{C}$ for $10 \mathrm{sec}$. QuantaSoft analysis software (Bio-Rad Laboratories) enabled abundance to be calculated for each sample.

\section{Case report}

A female underwent circulating tumor cell detection using SE-iFISH platform in the surgical oncology department, Quanzhou No.1 hospital, Fujian, China, in Dec 22, 2014. She was 56-year-old, during menopause, and has no family history of cancer. The results were $8 \mathrm{CTCs} / 7.5 \mathrm{ml}$ which indicated a high risk of cancer (Fig. 1). However, the levels of AFP, cancer antigen (CA)-125, CA19-9, carcinoembryonic antigen (CEA) and CA15-3 were in normal range. This patient also accepted an HPV typing (HPV16, 18, 31, 33, 35,39, 45, 51, 52, 56, 58, 59, 68, 6, 11, 42, 43, 44, 53, 66 and CP8304) test, and the results were negative. The thinprep cytology test showed no intraepithelial neoplasia. Written informed consent was obtained from this person for the present study.

To define the mutation spectrum of CTCs, we performed laser capture microdissection to isolate the CTCs, and whole genome amplification and whole exome capture DNA 
Table I. Cancer-related variations annotation in COSMIC database.

\begin{tabular}{|c|c|c|c|c|c|}
\hline Gene & Loci & Ref & Alt & Histology & Organ \\
\hline HOXB3 & 46629737 & $\mathrm{TG} / \mathrm{TG}$ & $\mathrm{TG} / \mathrm{T}$ & Adenocarcinoma & Colon \\
\hline CENPQ & 49459978 & $\mathrm{AA}$ & $\mathrm{AG}$ & Adenocarcinoma & Colon \\
\hline TSPAN10 & 79612161 & $\mathrm{CC}$ & $\mathrm{TT}$ & Adenocarcinoma & Colon \\
\hline TCEAL8 & 102508779 & GG & GT & Adenocarcinoma & Rectum \\
\hline MXRA5 & 3241256 & $\mathrm{TT}$ & $\mathrm{CC}$ & Adenocarcinoma & Colon \\
\hline PRR25 & 855717 & $\mathrm{CC}$ & GG & Adenocarcinoma & Rectum \\
\hline HCAR3 & 123200693 & AA & GG & Adenocarcinoma & Colon \\
\hline PARL & 183547404 & $\mathrm{CT} / \mathrm{CT}$ & $\mathrm{CT} / \mathrm{C}$ & Adenocarcinoma & Colon \\
\hline KDM6B & 7752901 & $\mathrm{GC} / \mathrm{GC}$ & $\mathrm{G} / \mathrm{G}$ & Adenocarcinoma & Colon \\
\hline DCST1 & 155019710 & $\mathrm{AA}$ & $\mathrm{CC}$ & Adenocarcinoma & Rectum \\
\hline RAI1 & 17696531 & GG & $\mathrm{CC}$ & Adenocarcinoma & Colon \\
\hline ITGA4 & 182347350 & GG & GA & Adenocarcinoma & Cecum \\
\hline ACKR2 & 42907112 & AA & $\mathrm{AC}$ & Adenocarcinoma & Cecum \\
\hline FCRLA & 161683136 & GG & $\mathrm{AA}$ & Adenocarcinoma & Stomach \\
\hline TNRC18 & 5396715 & $\mathrm{TT}$ & $\mathrm{CC}$ & Adenocarcinoma & Stomach \\
\hline ASAP1 & 131124559 & TT & $\mathrm{CC}$ & Adenocarcinoma & Stomach \\
\hline CCDC153 & 119063908 & $\mathrm{CC}$ & $\mathrm{TT}$ & Adenocarcinoma & Stomach \\
\hline OR10G7 & 123909627 & $\mathrm{TT}$ & $\mathrm{CC}$ & Adenocarcinoma & Stomach \\
\hline CLEC1B & 10149406 & TT & $\mathrm{CC}$ & Adenocarcinoma & Stomach \\
\hline BRCA1 & 41244000 & TT & $\mathrm{CC}$ & Adenocarcinoma & Stomach \\
\hline LGALS14 & 40199914 & $\mathrm{CC}$ & GG & Adenocarcinoma & Stomach \\
\hline CLC & 40225646 & GG & AA & Adenocarcinoma & Stomach \\
\hline CHGB & 5904040 & GG & AA & Adenocarcinoma & Stomach \\
\hline IGLL1 & 23915652 & GG & GA & Adenocarcinoma & Stomach \\
\hline GCNT2 & 10557242 & GA/GA & $\mathrm{GA} / \mathrm{G}$ & Adenocarcinoma & Stomach \\
\hline MINA & 97664725 & $\mathrm{CC}$ & $\mathrm{TT}$ & Cancer & Breast \\
\hline LINC01168, LOC100128127 & 134886618 & GG & $\mathrm{CC}$ & Cancer & Breast \\
\hline WDR90 & 701656 & $\mathrm{CC}$ & TT & Cancer & Breast \\
\hline CHTF18 & 840378 & AA & GG & Cancer & Breast \\
\hline ZNF286A & 15611495 & TT & $\mathrm{CC}$ & Cancer & Breast \\
\hline BPIFB3 & 31656632 & $\mathrm{CC}$ & $\mathrm{CG}$ & Cancer & Breast \\
\hline TNN & 175067689 & GG & $\mathrm{AA}$ & Squamous cell carcinoma & Skin, face \\
\hline MAN2B2 & 6602344 & GG & GT & Malignant melanoma & Skin, arm \\
\hline SMOC1 & 70420202 & GG & GA & Malignant melanoma & Skin \\
\hline SRSF4 & 29481412 & $\mathrm{CC}$ & AA & Hepatocellular carcinoma & Liver \\
\hline COL15A1 & 101778265 & GG & TT & Astrocytoma & Brain \\
\hline IDI2 & 1065491 & GG & TT & Renal clear cell carcinoma & Kidney \\
\hline KRTAP4-7 & 39240627 & $\mathrm{TT}$ & $\mathrm{TC}$ & Transitional cell carcinoma & Bladder \\
\hline LTBP4 & 41118056 & AA & GG & Meningioma & Meninges \\
\hline TGM3 & 2290333 & $\mathrm{CC}$ & $\mathrm{AA}$ & Adenocarcinoma & Lung \\
\hline UMODL1 & 43546494 & GG & GA & Endometrioid carcinoma & Endometrium \\
\hline TMEM37 & 120194651 & $\mathrm{~A} / \mathrm{A}$ & $\begin{array}{l}\text { AGTGTGC/ } \\
\text { AGTGTGC }\end{array}$ & Serous carcinoma & Ovary \\
\hline
\end{tabular}

COSMIC, catalogue of somatic mutations in cancer.

sequencing (WES) on the 8 CTCs from this female. Sequencing achieved $81.5 x$ mean coverage on targeted exons. 34215 SNVs and 6807 InDels were defined in the CTCs. Mapping these variations in COSMIC (http://cancer.sanger.ac.uk/cosmic/), 42 variations and InDels were correlated significantly with cancer (Table I). Notably, 13, 12 and 6 mutations were related to large intestinal cancer, gastric cancer and breast cancer, respectively (Fig. 2A). Fig. 2B shows 13 gene variations and their mutational frequency in large intestinal cancer, according to a TCGA study. 

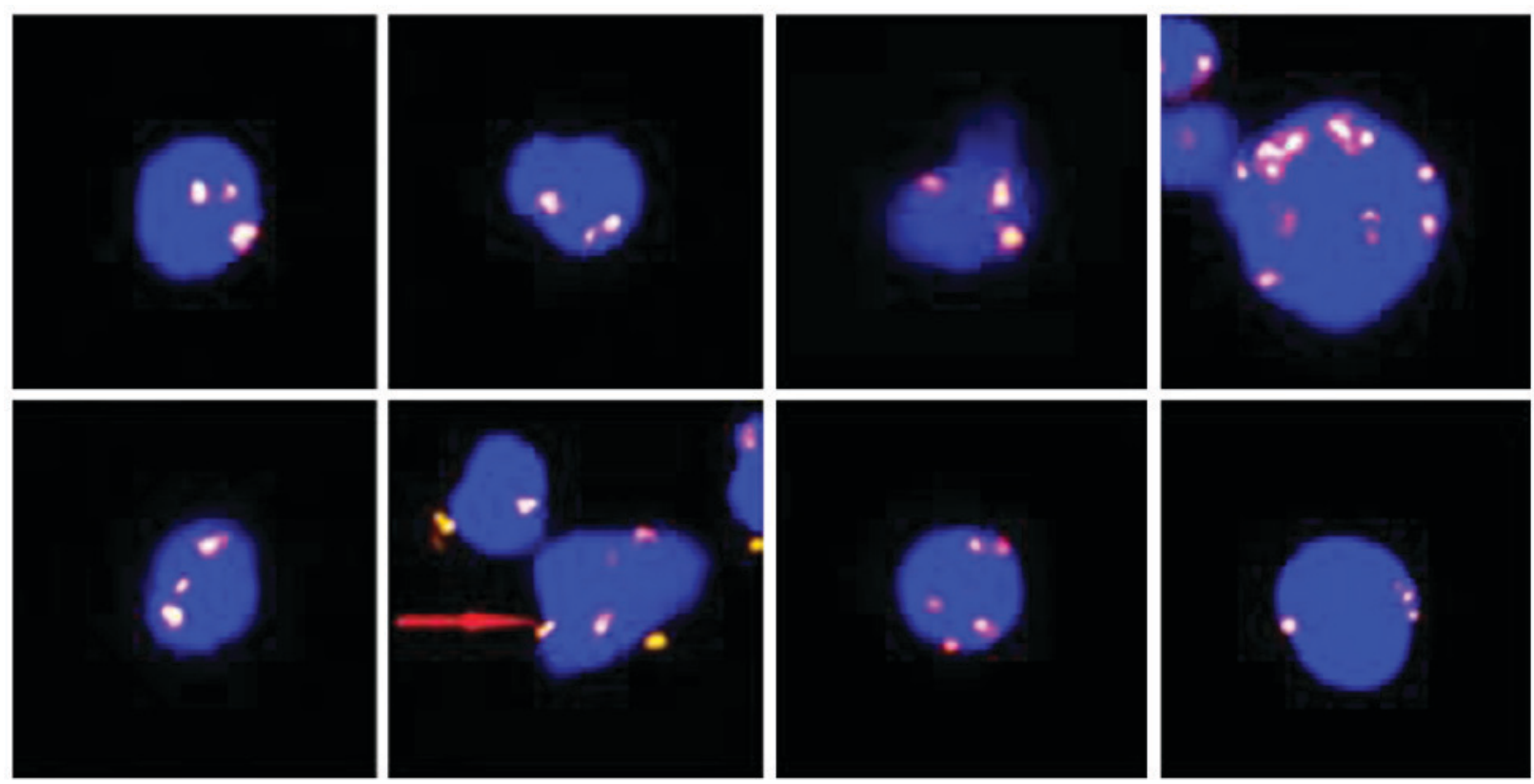

Figure 1. Eight polyploid CTCs (CK-/CD45-/DAPI+/CEP8+) identified by the SE-iFISH platform in one female. Magnification, x400. DAPI, blue; CEP8, orange; CK, green; CD45, red. Red arrow, CTC in this image is this polyploidy pointed by red arrow, rather than another diploid. CTCs, circulating tumor cells; CEP8, Centromere Probe 8; CK, cytokeratin; DAPI, 4',6-diamidino-2-phenylindole; SE-iFISH, immunostaining-fluorescence in situ hybridization.
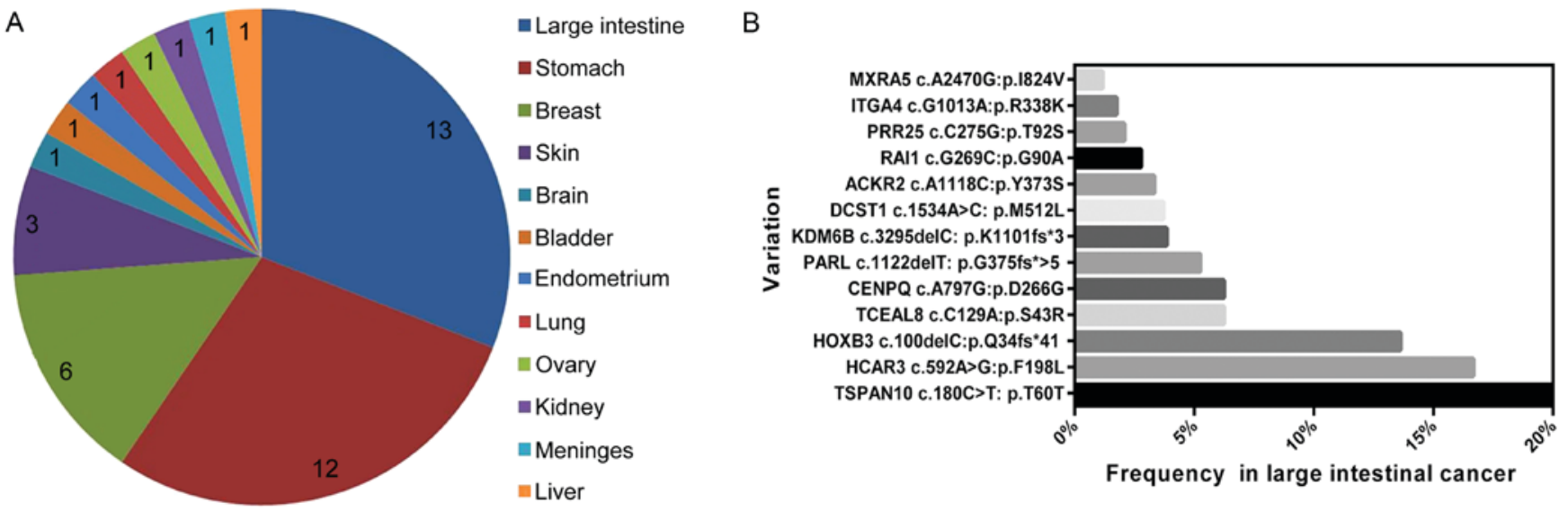

Figure 2. (A) Organ distribution of SNVs mapped to cancer. (B) Mutation frequency in large intestinal cancer of 13 variations, according to a TCGA study. SNVs, single nucleotide variation.

SNV annotation in COSMIC showed a high risk of large intestinal cancer, followed by gastric cancer and breast cancer. To check for lesions, the patient underwent a series of imaging examinations, including colonoscopy, gastroscopy and color Doppler ultrasound (in the thyroid gland, cervical lymph node, mammary gland and draining lymph nodes, liver, spleen, gallbladder, pancreas, uterus and adnexa, bladder and adjacent tissue).

A hemispherical polyp $(0.4 \mathrm{~cm})$ was observed in the sigmoid $18 \mathrm{~cm}$ away from the anus (Fig. 3A), which was then resected via endoscopic therapy. Pathological diagnosis showed that it was a hyperplastic polyp (Fig. 3B). No other lesions were detected in the stomach or breast by both gastroscopy and color Doppler ultrasound.

WES was performed on DNA of resected intestinal polyp, and achieved 107.71x mean coverage on targeted exons. But none of 13 SNVs in CTCs was found in polyp, which were related with large intestine cancer in COSMIC database. For higher sequencing depth, we analyzed these 13 SNVs in polyp's DNA using droplet digital PCR (ddPCR). ACKR2 c.A1118C, DCST1 c.A1534C, ITGA4 c.G1013A were positive in intestinal polyp (Fig. 4), other 10 SNVs were not detected.

CTCs in the peripheral blood of this patient were monitored after surgery, at 1 and 6 months during the follow-up. The number of CTCs reduced to $0 \mathrm{CTCs} / 7.5 \mathrm{ml}$, indicating a low risk of residual lesions (Fig. 5). And level of AFP, CA-125, CA19-9, CEA and CA15-3 in plasma were in normal range at 1 and 6 months during follow-up.

\section{Discussion}

CTCs in the blood have been suggested to be potential surrogate markers for minimal residual disease, and the precursor of metastatic disease (1). Their presence represents 

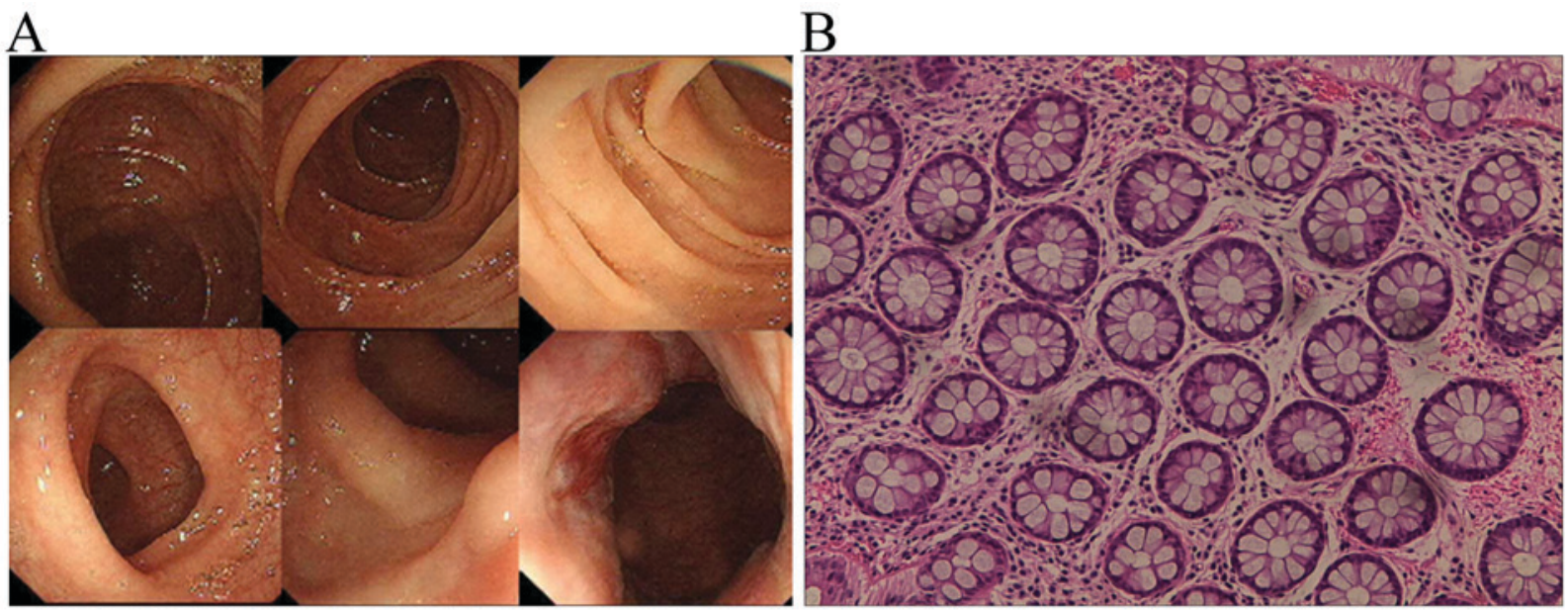

Figure 3. Imaging of the intestinal polyp via colonoscopy (A) and H\&E staining (B). Magnification, x400.
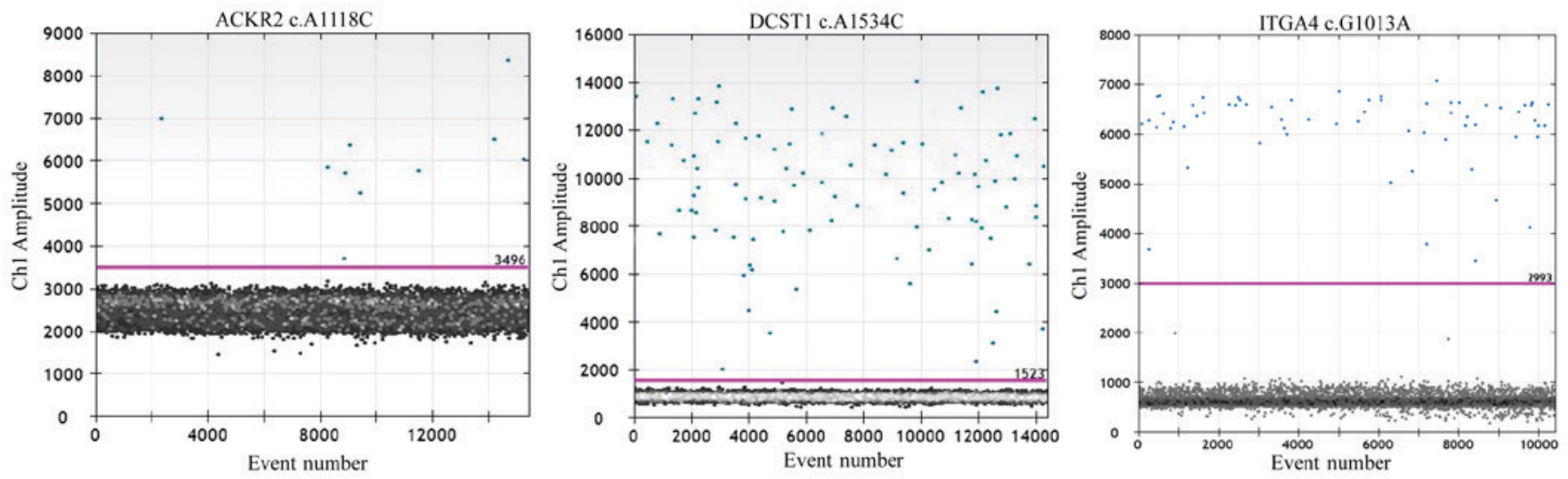

Figure 4. Fluorescence intensity droplets of ACKR2 c.A1118C, DCST1 c. A1534C, ITGA4 c.G1013A in intestinal polyp by ddPCR.

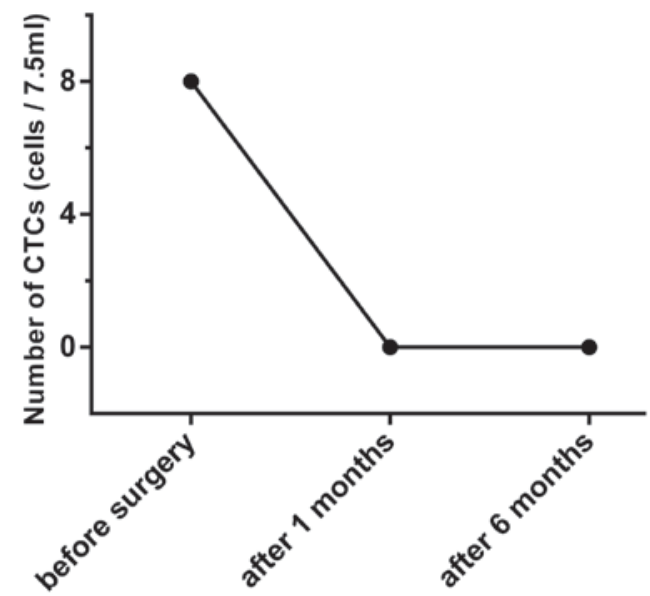

Figure 5. Comparison of CTCs before and after surgery in the patient. CTCs, circulating tumor cells.

an independent prognostic factor for reduced disease-free and overall survival $(3,4)$. The migration of CTCs seems to be an early event in human carcinogenesis, even before the cancer is visible in clinical imaging. CTCs have been detected in the blood of model animals when the tumor size is $<1 \mathrm{~mm}(6)$. The study of Ilie et al (11) showed that $\mathrm{CTC}^{+}$patients with chronic obstructive pulmonary disease (COPD) were all diagnosed with lung cancer during a 1-4 years follow up period. The search for CTCs, at present, may facilitate an early diagnosis of cancer or precancerosis.

Many studies have proven that the SE-iFISH platform (identified by CEP8, CK and CD45 (12)) has a higher CTC detection rate than the Cellsearch system $(7,8,10,13)$. The high detection rate of CTCs by the SE-iFISH platform was attributed to the following reasons, as previously reported (7). Firstly, the subtraction enrichment of the SE-iFISH platform uses immunomagnetic particles conjugated with anti-CD45 antibody to wipe off the WBCs, which doesn't depend on the EpCAM expression of CTCs; the expression of EpCAM on CTCs may decrease during epithelial-mesenchymal transition (EMT). Secondly, the SE-iFISH system not only identifies $\mathrm{CK}^{+}$CTCs by immunostaining, but also aneuploid CTCs by CEP8-fluorescence in situ hybridization; aneuploidy is a typical common cytogenetic abnormality malignant cells (14).

In this case, $8 \mathrm{CTCs} / 7.5 \mathrm{ml}$ were detected in one healthy female by $\mathrm{CK}-/ \mathrm{CD} 45-/ \mathrm{DAPI}+\mathrm{CEP}>2$, indicating a high risk of cancer. Via mapping of the CTC mutation spectrum in the COSMIC database, we proposed that the cells may have derived from large intestinal cancer, gastric cancer or breast cancer, or their precancerous lesions. A $0.4 \mathrm{~cm}$ hemispherical 
polyp was observed in the sigmoid, $18 \mathrm{~cm}$ away from the anus (Fig. 3A), which was diagnosed as a hyperplastic polyp by pathological determination (Fig. 3B). However, no lesions were found in the stomach or breast by gastroscopy and color Doppler ultrasound. There are small subsets of hyperplastic polyps with risk for development of colorectal cancer, although vast majority of hyperplastic polyps are innocent (15). There exists a degree of genetic and perhaps morphologic heterogeneity amongst hyperplastic polyps (16). It has been proposed that hyperplastic polyps may serve as the initial lesion in a serrated neoplasia pathway that results in the $15 \%$ sporadic colorectal adenocarcinomas that are microsatellite unstable (17-19).

A total of 13 SNVs above (Fig. 2B) weren't detected in resected intestinal polyp using WES, while three SNVs including ACKR2 c.A1118C, DCST1 c.A1534C, ITGA4 c.G1013A were detected by ddPCR (Fig. 4). That may be attributed to following reasons. Tumor evolve from benign to malignant lesions by acquiring a series of mutations over a long time. Genetic variations emerged in a minor fraction of a cell population before histology change during tumorigenesis (20). The cells with gene mutations may persist, but the cell numbers are very small compared with wild type cells. Sample for DNA extraction couldn't represent overall perspective of polyp because of heterogeneity. WES of hyperplastic polyp in this case achieved limited sequencing depth about 100x, which was hard to detect these rare mutations. While, ddPCR have a high degree of sensitivity, which is available for detecting $0.001 \%$ mutant fractions.

The CTC detection results were $0 \mathrm{CTC} / 7.5 \mathrm{ml}$ in this patient's blood at 1 and 6 months after removal of the intestinal polyp. The study by Wu et al (9) reported that a decline of the CTC count after surgery indicated better prognosis, while an increase indicated fast recurrence for colorectal cancer patients. From the results above, we can deduce that the intestinal polyp could have been the main source of the 8 CTCs in this patient. And this intestinal polyp had risk for development of early colorectal cancer.

Sporadic CRC is a somatic genetic disease that may be influenced by the local colonic environment and the individual's background genetic makeup (21). Patients often present after 60 years of age, with most cancers originating from precursor initiating adenomas that, over 1-2 decades, transform into cancer. Because tumor cell dissemination appears to be an early event in tumor progression, CTCs may appear at very early stages of tumor development. Genomic alterations occur during the initiation and progression of a normal colonic cell into a neoplastic and malignant cell. Changes in the nucleic acids of the cancer cell might be detected from blood circulation, such as CTCs (22). Early screening of this population at age 50 years or older is effective and sustainable, reducing mortality from CRC and decreasing the incidence of CRC. Preventive surgery for adenoma, polyps or cancer is a vital approach towards a cure (23).

There is few study focused on CTCs' organ derivation for person who isn't previously diagnosed with cancer using WES, although some studies performed whole-exome sequencing of CTCs on patients with cancer $(24,25)$. In summary, SE-iFISH CTC detection represents a potential tool for early stage cancer screening, and next generation sequencing of CTCs provides a window into the source of the CTCs and the properties of the solid tumor.

\section{Acknowledgements}

Not applicable.

\section{Funding}

The present study was supported by grants from Fujian Natural Science Foundation (grant nos. 2016J01612 and 2018J01198), and Fujian Medical Innovation Project (grant no. 2017-CX-47).

\section{Availability of data and materials}

The datasets generated during the current study are available from the corresponding author on reasonable request.

\section{Authors' contributions}

QXP and ZJS contributed to the study design; ZJS and JMZ were major contributors to writing the manuscript; ZJS interpreted the clinical data of patient; SYK, JZ and XYL collected samples and performed experiments; YW and JMZ analyzed the whole-exome sequencing data. QHS contributed to data analysis and manuscript revision.

\section{Ethics approval and consent to participate}

Ethical approval for the recruitment of human subjects was obtained from the Ethics Committee of First Hospital of Quanzhou Affiliated to Fujian Medical University and was consistent with ethical guidelines provided by the Declaration of Helsinki (1975). Written informed consent was obtained from each patient.

\section{Patient consent for publication}

All individuals whose data were used provided informed consent for publication.

\section{Competing interests}

The authors declare that they have no competing interests.

\section{References}

1. Yap TA, Lorente D, Omlin A, Olmos D and de Bono JS: Circulating tumor cells: A multifunctional biomarker. Clin Cancer Res 20: 2553-2568, 2014.

2. Magbanua MJ, Carey LA, DeLuca A, Hwang J, Scott JH, Rimawi MF, Mayer EL, Marcom PK, Liu MC, Esteva FJ, et al: Circulating tumor cell analysis in metastatic triple-negative breast cancers. Clin Cancer Res 21: 1098-1105, 2015.

3. CristofanilliM,BuddGT,Ellis MJ,StopeckA,Matera J,MillerMC, Reuben JM, Doyle GV, Allard WJ, Terstappen LW and Hayes DF: Circulating tumor cells, disease progression, and survival in metastatic breast cancer. N Engl J Med 351: 781-791, 2004

4. Cohen SJ, Punt CJ, Iannotti N, Saidman BH, Sabbath KD, Gabrail NY, Picus J, Morse M, Mitchell E, Miller MC, et al: Relationship of circulating tumor cells to tumor response, progression-free survival, and overall survival in patients with metastatic colorectal cancer. J Clin Oncol 26: 3213-3221, 2008 . 
5. Scher HI, Heller G, Molina A, Attard G, Danila DC, Jia X, Peng W, Sandhu SK, Olmos D, Riisnaes R, et al: Circulating tumor cell biomarker panel as an individual-level surrogate for survival in metastatic castration-resistant prostate cancer. J Clin Oncol 33: 1348-1355, 2015.

6. Rhim AD, Mirek ET, Aiello NM, Maitra A, Bailey JM, McAllister F, Reichert M, Beatty GL, Rustgi AK, Vonderheide RH, et al: EMT and dissemination precede pancreatic tumor formation. Cell 148: 349-361, 2012.

7. Sheng Y, Wang T, Li H, Zhang Z, Chen J, He C, Li Y, Lv Y, Zhang J, Xu C, et al: Comparison of analytic performances of Cellsearch and iFISH approach in detecting circulating tumor cells. Oncotarget 8: 8801-8806, 2017.

8. Gao Y, Zhu Y, Zhang Z, Zhang C, Huang X and Yuan Z: Clinical significance of pancreatic circulating tumor cells using combined negative enrichment and immunostaining-fluorescence in situ hybridization. J Exp Clin Cancer Res 35: 66, 2016.

9. Wu W, Zhang Z, Gao XH, Shen Z, Jing Y, Lu H, Li H, Yang X, Cui X, Li Y, et al: Clinical significance of detecting circulating tumor cells in colorectal cancer using subtraction enrichment and immunostaining-fluorescence in situ hybridization (SE-iFISH). Oncotarget 8: 21639-21649, 2017.

10. Li Y, Zhang X, Ge S, Gao J, Gong J, Lu M, Zhang Q, Cao Y, Wang DD, Lin PP and Shen L: Clinical significance of phenotyping and karyotyping of circulating tumor cells in patients with advanced gastric cancer. Oncotarget 5: 6594-6602, 2014

11. Ilie M, Hofman V, Long-Mira E, Selva E, Vignaud JM Padovani B, Mouroux J, Marquette CH and Hofman P: 'Sentinel' circulating tumor cells allow early diagnosis of lung cancer in patients with chronic obstructive pulmonary disease. PLoS One 9: e111597, 2014.

12. Ge F, Zhang H, Wang DD, Li L and Lin PP: Enhanced detection and comprehensive in situ phenotypic characterization of circulating and disseminated heteroploid epithelial and glioma tumor cells. Oncotarget 6: 27049-27064, 2015.

13. Zhang Y, Wang F, Ning N, Chen Q, Yang Z, Guo Y, Xu D, Zhang D, Zhan T and Cui W: Patterns of circulating tumor cells identified by CEP8, CK and CD45 in pancreatic cancer. Int J Cancer 136: 1228-1233, 2015.

14. Zhang J, Li S, Liu F, Zhou L, Shao N and Zhao X: SELEX aptamer used as a probe to detect circulating tumor cells in peripheral blood of pancreatic cancer patients. PLoS One 10 : e0121920, 2015
15. Hyman NH, Anderson P and Blasyk H: Hyperplastic polyposis and the risk of colorectal cancer. Dis Colon Rectum 47: 2101-2104,2004.

16. Goldstein NS, Bhanot P, Odish E and Hunter S: Hyperplastic-like colon polyps that preceded microsatellite-unstable adenocarcinomas. Am J Clin Pathol 119: 778-796, 2003.

17. Jass JR, Young J and Leggett BA: Hyperplastic polyps and DNA microsatellite unstable cancers of the colorectum. Histopathology 37: 295-301, 2000.

18. Jass JR: Serrated route to colorectal cancer: Back street or super highway? J Pathol 193: 283-285, 2001.

19. Iino H, Jass JR, Simms LA, Young J, Leggett B, Ajioka Y and Watanabe H: DNA microsatellite instability in hyperplastic polyps, serrated adenomas, and mixed polyps: A mild mutator pathway for colorectal cancer? J Clin Pathol 52: $5-9,1999$.

20. Vogelstein B, Papadopoulos N, Velculescu VE, Zhou S, Diaz LA Jr and Kinzler KW: Cancer genome landscapes. Science 339: 1546-1558, 2013.

21. Carethers JM and Jung BH: Genetics and genetic biomarkers in sporadic colorectal cancer. Gastroenterology 149: 1177-1190.e3, 2015.

22. Krebs MG, Metcalf RL, Carter L, Brady G, Blackhall FH and Dive C: Molecular analysis of circulating tumour cells-biology and biomarkers. Nat Rev Clin Oncol 11: 129-144, 2014.

23. Shaukat A, Mongin SJ, Geisser MS, Lederle FA, Bond JH, Mandel JS and Church TR: Long-term mortality after screening for colorectal cancer. N Engl J Med 369: 1106-1114, 2013.

24. Lohr JG, Adalsteinsson VA, Cibulskis K, Choudhury AD, Rosenberg M, Cruz-Gordillo P, Francis JM, Zhang CZ, Shalek AK, Satija R, et al: Whole-exome sequencing of circulating tumor cells provides a window into metastatic prostate cancer. Nat Biotechnol 32: 479-484, 2014.

25. Faugeroux V, Lefebvre C, Pailler E, Pierron V, Billiot F, Marcaaillou C, Tourlet S, Vielh P, Dogan S, Rameau P, et al: Whole-exome sequencing of single circulating tumor cells is a useful tool for studying the intrapatient genetic heterogeneity in metastatic prostate cancer. J Clin Oncol 35 (6 Suppl): S148, 2017.

(i) $(2)$ This work is licensed under a Creative Commons Attribution-NonCommercial-NoDerivatives 4.0 International (CC BY-NC-ND 4.0) License. 